\title{
On Alleviation of New User Problem in Collaborative Filtering using SNA Theory
}

\author{
Yang Yujie ${ }^{1}$, Zhang Huizhi ${ }^{1}$ and Wang Xianfang ${ }^{1}$ \\ ${ }^{1}$ Institute of Computer and Information Technology, Henan Normal University, \\ Computational Intelligence and Data Mining Engineering Technology Research \\ Center of Colleges and Universities in Henan Province, Xinxiang Henan China \\ yujieyangyujie@gmail.com,zhanghuizhi0418@gmail.com,xfw1969@hotmail.com
}

\begin{abstract}
Collaborative filtering is the most used personalized recommendation technology. However, the traditional collaborative filtering faces the cold start problem and data sparsity, which deteriorates user experience and reduces the prediction accuracy. This paper presents a novel solution of new user problem with social network analysis (SNA) theory. First, the user relationship network is built based on the user-item rating matrix. Then all users will be divided into many different cliques according to SNA. The demographic user information of new user is used to find similar users in user attributes. Further, the candidate neighbors of new user can be obtained from cliques. Then, the preferential attachment characteristic of the free-scale network is introduced to eliminating the unsuitable neighbors. Finally, items are recommended with user-based nearest neighbor recommendation algorithm. The experiments show that the proposed approach alleviates the new user problem and improves the prediction accuracy effectively compared with other algorithms.
\end{abstract}

Keywords: New User Problem, Cold Start Problem, Collaborative Filtering, Social Network Analysis, Cliques, Preferential Attachment Characteristic

\section{Introduction}

Collaborative filtering (CF) has becoming more and more efficient solution to overcome "information overload" and to provide personalized services. Generally speaking, collaborative filtering can be categorized into memory-based and model-based [1]. The CF has been applied many areas successfully, such as book sites, movie sites and some other Ecommerce sites [2]. However, the CF also suffers from many issues, for instance, the ability to handle data sparsity, cold start problems and scalability.

Cold start problem includes new user problem and new item problem. The former is how recommend items to users who have not rated any items. The latter is how recommend items which have not been rated to users. This paper aims to solve the new user problem of the cold start problems in the collaborative filtering. There are many researches have presented some approaches to solve the new user problem [3-5].

The difficulty in new user problem is how to find the neighbors of new users effectively. Unlike many other methods, the social network analysis theories are used in the paper. First, the similarities among existing users are computed according to the user-item rating matrix. The user relationship network will be built based on these similarities. According to social network analysis theory [6], the network will be divided into many cliques which compose of many users with similar interests. User information vector model which is derived from the users' registration information will be used to measure the similarity between new users and 
existing users. Then, candidate neighbors set can be found combined with the cliques. The preferential attachment characteristic of the BA scale free network model [7] is used to eliminate the dissimilar neighbors from the candidate neighbors set. Finally, the traditional user-based nearest neighbor algorithm is used to recommend items for new users. And the experiments show the improving performance.

In the remainder of this paper, related work on the state-of-art of cold start solutions is made; then a novel approach for alleviating new user problem will be explained in detail; several performance evaluation rules are introduced to measure the proposed method; it is then shown how the proposed algorithm is applied to the data set of MovieLens and the experiments are described and the results analyzed; finally, it gives the conclusion.

\section{Related Work}

New user problem is that user has not rated any items yet in the recommender systems. It often occurs when the user enter into the recommender systems firstly, which leads to bad user experience and low customer loyalty. There are many researchers who have focused on new user problem and proposed some solutions.

The most commonly-used approaches are by means of additional information, just as browsing time, click, and interaction with users and so on. [3] proposed dynamic browsing tree model to solve the new item problem. This method first makes the user browsing records to transform to dynamic browsing tree based on product categories of E-commerce website. Then it designs a fresh degree decay operator based on access time. New item will be chosen based on the matching degree between new item and dynamic browsing trees of all users. The implicit information of new users and multi-attribute rating matrix is proposed to solve the cold start problem [8]. This method first collects implicit information and combines other rating information to create a user-item rating matrix. Then user-item attribute rating matrix and user attribute item raring matrix will be built.

Hybrid approaches are another most used for solving cold start problem. [4] proposed a novel hybrid recommendation approach to address the cold start problem. This method makes use of cross-level association rules to integrate content information about domain items into collaborative filters. Hybrid model [9] is developed based on the analysis of two probabilistic aspect models using pure collaborative filtering to combine with users' information. [10] proposed a hybrid algorithm by using both the ratings and content information to tackle itemside cold-start problem. The method clusters items based on the rating matrix and utilize the clustering results and item content information to build a decision tree to associate the novel items with the existing ones.

Moreover, rule-based induction [5] on cold user data has been also used to improve new user recommendation. It combines with various-community spaces which relate to cold user data (age, occupation, location, etc.). [11] evaluates the performance of different collaborative algorithms in cold-start situations. Its aim is to try to provide guidelines to help in the selection and tuning of different state-of-the-art collaborative algorithms in different stages of the recommender systems life-cycle. Its analysis shows that item-based algorithms perform better with respect to singular value decomposition based ones in the early stage of the coldstart problem. Clustered social ranking [12] is a novel search and recommendation technique specifically developed to support new users of Web 2.0 websites finding content of interest. [13] combines genetic algorithms with collaborative filtering to alleviate cold start problem.

With the rapid development of online social network, the social network analysis theory is also used to recommender systems. The social network-based recommender systems (SNRSs) $[14,15]$ which integrate social network analysis theory in recommender systems to improve 
their recommendation. [16] Presented a model which assists users in finding candidates who can belong to their social network. This model not only makes easier the use of SNRSs, but also encourages the use of the embedded social network. [17] introduces various social network analysis methods. [18] discusses the trend of recommender systems that are based on network analysis. It presents a framework of recommendations based on information network analysis.

However, there are few papers to apply social network analysis to deal with cold start problem. In this paper, our research work provides an efficient solution to address the cold start problem in collaborative filtering by means of social network analysis theories.

\section{Proposed Approach}

\subsection{User Relationship Network and Cliques}

In collaborative filtering systems, two users are thought to have a certain relationship if they have rated the same items at least one time. Generally speaking, a network is composed of nodes and the relationships among nodes. Formally, let us consider a network as a graph $G=(U, E)$ in which $U$ represents nodes and $E$ represents links. In this paper, the nodes represent the users and, the relations denote the similarities among users, that are links. In this paper, the building network is undirected weighted network. The weights can be calculated from the similarities among users.

According to SNA, cliques are some sub-structures of the network. From the view of social structure, clique focuses attention on how solidarity and connection of social network. The general definition of a clique is simply a sub-set of nodes which are more closely tied to each other than they are to nodes which are not part of the group[18]. More accurately, it insists that every member have a direct tie with each and every other member. In our approach, the existing users will be divided into many cliques.

In order to build the user relationship network, firstly, we need compute the similarity among each pair users. Assume that $U=\left\{u_{1}, u_{2}, \cdots u_{N}\right\}$ denotes the set of users, $P=\left\{p_{1}, p_{2}, \cdots, p_{M}\right\}$ for the set of items, and $R$ as an $N \times M$ matrix of ratings $r_{i, j}$, with $i \in 1, \cdots, N, j \in 1, \cdots, M$. There are many algorithms to determine the similarity among users: Pearson's correlation coefficient, cosine similarity, and adjusted cosine measure[19] and so on. In the paper, Pearson's correlation coefficient is used. So, the similarity between user $u_{i}$ and $u_{j}$ is as follows,

$$
\operatorname{sim}\left(u_{i}, u_{j}\right)=\frac{\sum_{p \in P_{u_{i}} \cap P_{u_{j}}}\left[\left(r_{i, p}-\overline{r_{i}}\right) *\left(r_{j, p}-\overline{r_{j}}\right)\right]}{\sqrt{\sum_{p \in P_{u_{i}} \cap P_{u_{j}}}\left(r_{i, p}-\overline{r_{i}}\right)^{2} *\left(r_{j, p}-\overline{r_{j}}\right)^{2}}}
$$

where $\overline{r_{i}}$ and $\overline{r_{j}}$ corresponds to the average rating of user $u_{i}$ and $u_{j}$ respectively. $P_{u_{i}}$ denotes the item set of items rated by user $u_{i} . P_{u_{j}}$ is the item set of user $u_{j}$ rating. In practice, because the amount of items is very large, users may only rate few items. The number of overlapping item among users may be very few. This leads to the inaccurate similarity. For more accuracy of the similarity, a parameter $\gamma$ which denotes the number of 
overlapping rating between two users will be added to adjust. So, the improved formula is as follows,

$$
\operatorname{sim}^{\prime}\left(u_{i}, u_{j}\right)=\left\{\begin{array}{l}
\operatorname{sim}\left(u_{i}, u_{j}\right), \gamma \geq T \\
-1, \gamma<T
\end{array}\right.
$$

where $T$ is a threshold, the more value, the more accuracy of the similarity.

Then user relation network will be built based on these similarities. The network which consists of all users will be divided many cliques. This network is an undirected graph, in which edges are not directed. For making cliques, it needs binaries. The links are set 1 if the similarity greater or equal a solid value, otherwise 0. After that, UCINET [17] will be used to divide the network into many cliques. So, users who have similar interests and preferences will be divided into the same cliques [20].

Compared to k-means cluster algorithm [10], clique has many advantages. On one hand, kmeans cluster divides the similar users into the same cluster, however for one user, he/she is divided only one cluster. Intuitively, many users will have all kind of interests and join different communities. So, the user should belong to several clusters. Cliques can avoid this obstacle. On other hand, k-means algorithm requires the $k$ less $n$, which $k$ denotes the number of clusters, $n$ is the number of users. In fact, the cluster number may be more than that of the users. However, cliques number can more than users. Finally, clique can also present the user relation better.

\subsection{User Information Vector Model}

For the cold user, there is not any rating to compute the similarity with the exiting users. However, registration information of new users can be used. First, the user information vector model is presented. Then, the candidate neighbor set can obtain through this model.

Assume that $A=\left[a_{1}, a_{2}, \cdots, a_{n}\right]$ denotes the user information vector, $a_{i}$ is the $i$ th attribution. The attribution includes age, occupation, and sex and so on. For instance, user $u_{k}$ can be represented $A_{k}=[26$, doctor,male $]$. Based on these information vectors, the similarity can be computed between new users and existing users. If the weighting is $\omega_{k}$ for the attribution $a_{k}$, the similarity formula is as follows,

$$
\operatorname{sim}^{\prime \prime}\left(u_{\text {new }}, u_{\text {old }}\right)=\sum_{k=1}^{n} \omega_{k} \text {, if } a_{\text {newk }}=a_{\text {old }}
$$

where $a_{\text {newk }}$ and $a_{\text {oldk }}$ represent the $k$ attribution of user $u_{\text {new }}$ and $u_{\text {old }}$ respectively. Intuitively, the weight of each attribution is not same. It should set value based on the ability of distinguishing different users. In this paper, Shannon formula is used to measure the abilities of different attributions. Assume the number of item types is $k, p_{k}$ is the probability of the $k$ type, when class the items in accordance with the ith attribution. So, the weight of the ith attribution is:

$$
\omega_{i}=-\frac{1}{\sum_{k=1}^{K}\left(p_{k} * \log _{2} p_{k}\right)}
$$


According to the information theory, the greater the amount of information, the more random users is distinguished. The ability of distinguishing different users is lower correspondingly. So, the weight of attribution is set the reciprocal of the amount of information.

Through computing the user information similarity based on the user information vector model, it can get a user set $B$ in which is the most similar with the new users' information. Then, candidate neighbor set $B^{\prime}$ which includes all the users who are in the same cliques with $B$.

\subsection{Select Neighbors}

Because the ability of distinguishing different users by user information is low, there are many pseudo-neighbors in the set $B^{\prime}$. This will decrease the prediction accuracy of the new users. So, some measures should be adopted to eliminate those pseudo-neighbors.

In real life, there are many networks which belong to the BA scale free network model [7]. That is, the network degree distribution follows the power law distribution. For instance, Internet and WWW, movie actor network, food chain network and so on. Preferential attachment characteristic is a main feature in BA scale free network. The preferential attachment characteristic is that the new node is tend to link to the nodes which have large degree. This phenomenon is also known as rich get richer or Matthew effect. In the experiment of this paper, the network consists of existing users which also belongs to BA network. Figure 1 shows the network degree distribution. The power law coefficient is about 1.22. Another property of BA network is that it has larger clustering coefficient and smaller average distance [21]. So, we can also compute the clustering coefficient and average distance of the network. In our experiment, the clustering coefficient and average distance of the user relation network is 0.036 and 3.177 respectively. From Figure 1 and these two properties, we can know that the user relation network is a BA scale free network.

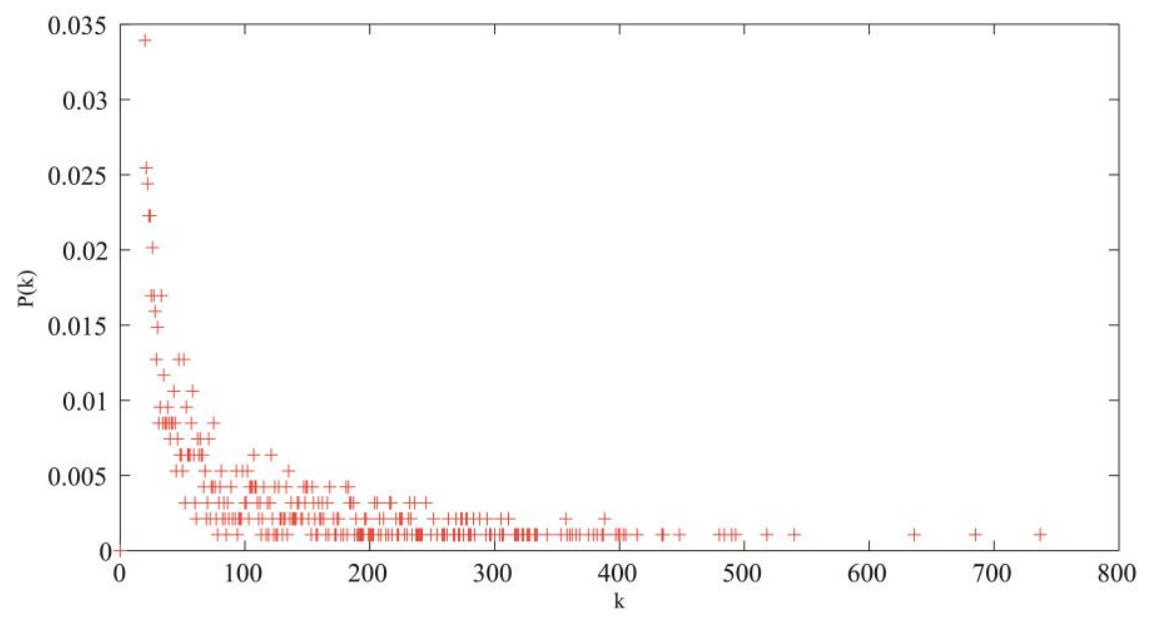

Figure 1. Degree Distribution of User Relation Network, $k$ Denotes the Degree of Node and $P(k)$ is the Probability for each Degree, the Power Law Coefficient is about 1.22

So, in this paper, the preferential attachment characteristic is applied to eliminate the pseudo-neighbors from the set $B^{\prime}$. Assume $\left|B^{\prime}\right|$ denotes the amount of element in $B^{\prime}$. First, the set $B^{\prime}$ is sorted by the degree of nodes (that is the number of similar users). Then, a certain 
percentage $\beta$ of the element is fetched from $B^{\prime}$ as the final neighbor set $B^{\prime \prime}$. That is $\left|B^{\prime \prime}\right|=\beta^{*}\left|B^{\prime}\right|$.

Then, user-based nearest neighbor recommendation is used to predict the items to new users. For the new user $u_{\text {new }}$, the prediction value of item $p \in P$ can be computed as follows,

$$
\operatorname{pre}\left(u_{n e w}, p\right)=\frac{\sum_{i=1}^{\left|B^{\prime \prime}\right|} r_{i, p}}{\left|B^{\prime \prime}\right|}
$$

where $|B "|$ is the number of set $B^{\prime \prime}, r_{i, p}$ is the rating of user $u_{i} \in B$ " to item. The prediction values are ranked in a descending order. The TopN items will be recommended to new user $u_{\text {new. }}$.

The whole step of the proposed approach is as follows:

1) Computing the similarities among users according to the user-item rating matrix, then building user relation network based on these similarities, and dividing the network into many cliques.

2) Defining the user information vector model and computing user similarities, the most similar users will be formed the candidate neighbor set $B^{\prime}$ combined with cliques.

3) Preferential attachment characteristic is applied to select the final nearest neighbor set $B^{\prime \prime}$.

4) Finally, user-based nearest neighbor recommendation is used to predict items for new users.

\section{Experiments and Analysis}

\subsection{Dataset}

Tphe MovieLens (http://www.movielens.umn.edu) dataset is used in this paper. In MovieLens, there are 100,000 ratings with 943 persons and 1682 movies. And each person had rated at least 20 movies. The user information includes age, sex, and occupation and so on. The movie includes 19 types.

The experiments uses n-fold cross validation. First, the dataset is divided into two parts, $20 \%$ of all persons are selected to be testing set, and the remaining as training set. In order to better evaluate the performance of new approach, we take the testing users from dataset uniformly. That is, the degree of all users is firstly computed and sorted by order. Then, the testing set is selected by equal intervals. So, the testing set includes all kinds of new users. For the training set, first, the similarities among each pair users will be computed in according to equation (1) and (2). In order to improve the accuracy, in the experiment, the parameter $T$ will be set as 5 in equation (2). Each user represents one node, and similarities form the relations among the user network. Finally, the cliques are obtained through UCINET.

For determining the nearest neighbor set of new user, the user information similarities are computed in according to equation (3) and (4). Then, the set $B^{\prime}$ can be obtained combined with the cliques and the set $B^{\prime \prime}$ further can be formed according to preferential attachment characteristic. Finally, the neighbor set of new user is formed according to the equation (5). 
In accpording to the neighbor set of new user, the traditional user-based nearest neighbor recommendation is used to predict and, the $T o p N$ of the ranking list will be recommended to the new users.

\subsection{Performance Evaluation}

In order to estimate the performance of the proposed approach, the precision of prediction is measured with three different metrics.

Recall. The recall score is the average proportion of items from test set that appear among TopN of the ranked list from the training set [22]. This measure should be as high as possible for good performance. Assume $N$ is the number of items which are in the testing set and liked by users, $n$ is the amount of items which the new user likes and appears in the recommended list. So, the recall is computed as follows,

$$
\text { Recall }=\frac{n}{N}
$$

Precision. The precision is the proportion of recommended items that the new users actually liked in the test set [4]. This measure is also as high as possible for good performance. The precision is computed as follows,

$$
\text { Precision }=\frac{n}{\operatorname{TopN}}
$$

F-measure. It is also known as the $F_{1}$ measure, which combines precision and recall into a single metric by taking the harmonic mean of them[4]. So, the F-measure is computed as follows:

$$
F_{1}=\frac{(2 * \text { Recall } * \text { Precision })}{(\text { Recall }+ \text { Precision })}
$$

\subsection{Results and Analysis}

For building the network, first, through Pearson's correlation coefficient algorithm, each pair users' similarity will be computed. Then binary similarities are constructed as follow: we set a relation threshold $R_{T}$, similarity values equal to $R_{T}$ or greater than $R_{T}$ are set 1 , that is, the user pair has link, otherwise 0 . In this experiment, we set $R_{T}$ as 0.75 and finally obtain 586 cliques through UCINET.

For finding the neighbors of new users, we consider different user attribution has different ability of distinguishing different users. Even the same attribution, the ability of distinguishing different users may not same. In MovieLens dataset, user attribution includes age, sex, occupation, position. In our experiment, we only use the first three attributions. So, the user information vector model is represented $A=\{$ age,sex,occupation $\}$. Table 1 , Table 2 and Table 3 give the ability of distinguishing different users in age, sex and occupation respectively. For better observing the relation between $\beta$ and the accuracy of recommendation, we set the $T o p N$ as 60 . The values are computed in according to equation (3). The three tables show the different ability of distinguishing different users. 
Table 1. The Ability of Distinguishing Users in Age

\begin{tabular}{|c|c|c|c|c|}
\hline age & $<18$ & $18 \sim 24$ & $25 \sim 34$ & $35 \sim 44$ \\
\hline weight & 0.2899 & 0.2814 & 0.2793 & 0.2821 \\
\hline Age & $45 \sim 49$ & $50 \sim 55$ & $>55$ & \\
\hline weight & 0.2794 & 0.2847 & 0.2789 & \\
\hline
\end{tabular}

Table 2. The Ability of Distinguishing Users in Sex

\begin{tabular}{|c|c|c|}
\hline sex & male & female \\
\hline weight & 0.2796 & 0.2814 \\
\hline
\end{tabular}

For new user problem, the most difficult problem is how to find suitable neighbor set. In order to obtain the neighbor set $B^{\prime \prime}$ of new user, the parameter $\beta$ plays an important role. So, the suitabple value should be studied. Figure 2 shows the performance curves with different $\beta$. The red start curve represents the trend of recall score with different $\beta$. From the curve, the recall increases gradually with $\beta$. It almost does not change when $\beta$ greater 0.7 . The green triangle is precision and the blue circle curve is F-measure. These two curves increase gradually with the increase of $\beta$. Generally speaking, $\beta$ should be range from 0.6 to 0.8 .

Table 3. The Ability of Distinguishing Users in Occupation

\begin{tabular}{|c|c|c|c|c|}
\hline occupation & educator & artist & adminis & student \\
\hline weight & 0.2796 & 0.2814 & 0.2854 & 0.2837 \\
\hline $\begin{array}{c}\text { occupation } \\
\text { weight }\end{array}$ & 0.2838 & 0.2824 & 0.2854 & 0.3368 \\
\hline occupation & programmer & retired & salesman & scientist \\
\hline weight & 0.2788 & 0.2830 & 0.2817 & 0.2797 \\
\hline occupation & technician & writer & librarian & other \\
\hline weight & 0.2834 & 0.2791 & 0.2954 & 0.2834 \\
\hline
\end{tabular}

Figure 3 shows the performance curves with different TopN. The red star curve represents thpe trend of recall score. From the trend we can see that the recall score increases gradually with the increase of the number of TopN. The green triangle curve denotes the trend of precision score. The curve shows that the precision decreases gradually with the increase of the number of TopN. This demonstrates that the recall score and precision are conflicting metrics. According to actual requires compromise between recall and precision is necessary. The blue circle curve is the trend of F-measure. It shows that the F-measure increases gradually with the increase of TopN.

[23] Presented transitive associations and spreading activation algorithms to solve the new user problem. Compared with [23], the proposed approach has a large improvement in recall, 
precision and F-measure. Because the recall and precision are conflicting metrics, and from the Figure 3 we suggest that TopN should be range from 40 to 80 .

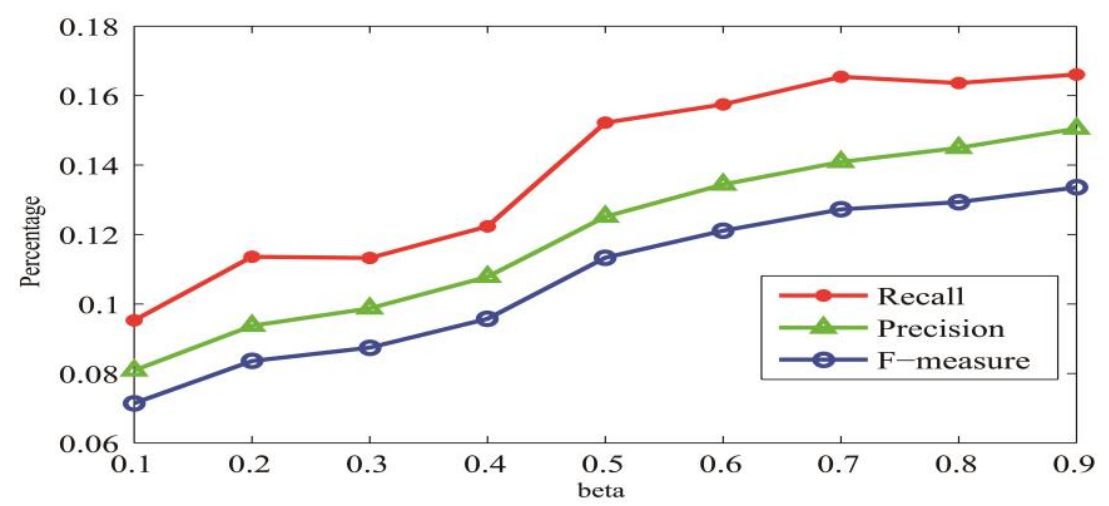

Figure 2. The Performances of Proposed Recommendation Approach with Different $\beta$, the Dotted Curve, Triangle Curve and Circle Curve Represent Recall, Precision and F-measure Respectively. TopN is Set 60

In time performance, the computing of similarities between each pair users, the building of user relation network and dividing cliques can be implemented offline. So, its speed is almost at the same level compared with the traditional recommender systems.

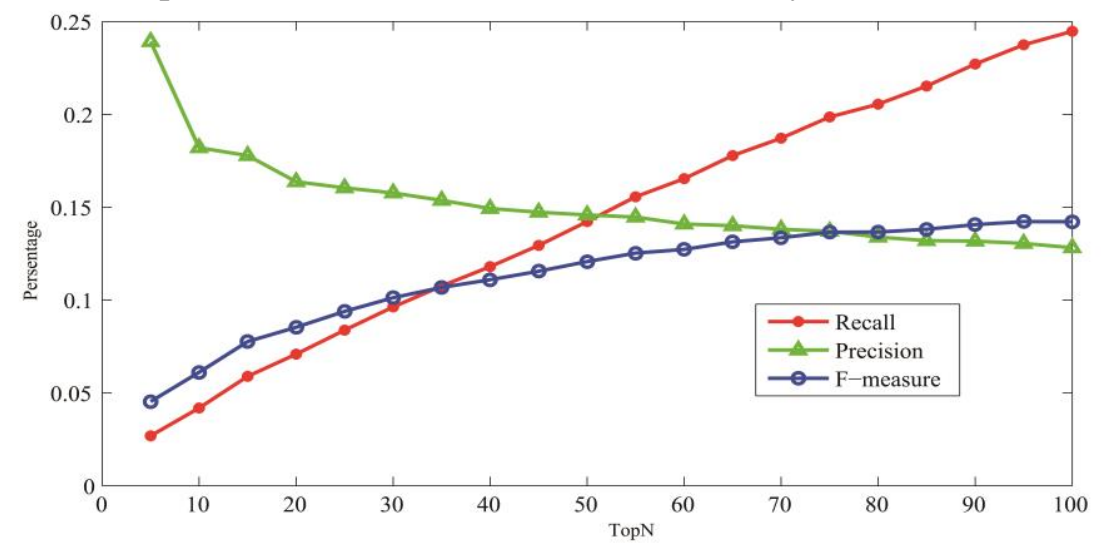

Figure 3. The Performances of Proposed Recommendation Approach with Different TopN, and $\beta$ is 0.7. The Dotted Curve, Triangle Curve and Circle Curve Represent Recall, Precision and F-measure Respectively

\section{Conclusions}

This paper proposes a novel approach to solve the new user problem in collaborative filtering based on the social network analysis theory. First, we compute the similarities between each pair users and build user relation network. Then cliques are divided from the network by using the UCINET software. For measuring the similarity between new users and existing users, the papper gives the ability of user information, which can distinguish different users. According to preferential attachment characteristic of the BA scale free network model and the cliques, the nearest neighbor of the new user can be selected. Finally, user-based nearest neighbor recommendation is used to recommend items to new users. The experiments show that the proposed approach can effectively alleviate the new user problem. In fact, the 
approach is not only suitable to new user problem, but also new item problem, which need only build the network of items, and compute the similarity between new item and existing items.

\section{Acknowledgements}

This work has been funded by the National Nature Science Foundation of China (No.61173071), the Foundation Cutting-edge Technologies in the Research Project of Henan province (No.132300410390) and the Youth Science Foundation of Henan Normal University (No.2012QK23)

\section{References}

[1] G. Adomavicius and A. Tuzhilin, "Toward the next generation of recommender systems: A survey of the state-of-the-art and possible extensions", IEEE Transactions on Knowledge and Data Engineering, Minneapolis, USA, (2005) June.

[2] G. Linden, B. Smith and J. York, "Amazon, Com recommendations: Item-to-item collaborative filtering", Internet Computing, IEEE, vol. 7, no. 1, (2003).

[3] C. Li, L. Ma, and K. Dong, "Collaborative filtering cold-start recommendation based on dynamic browsing tree model in e-commerce", Web Information Systems and Mining, WISM 2009, International Conference on, IEEE, Shanghai, China, (2009) November 7-8.

[4] C. Leung, S. Chan and F. Chung, "An empirical study of a cross-level association rule mining approach to cold-start recommendations", Knowledge-Based Systems, vol. 21, no. 7, (2008).

[5] A. Nguyen, N. Denos and C. Berrut, "Improving new user recommendations with rule-based induction on cold user data", Proceedings of the 2007 ACM conference on Recommender systems, ACM, Minnesota, USA, (2007) October 19-20.

[6] L. Pan and E. Santos, "An anytime-anywhere approach for maximal clique enumeration in social network analysis", Systems, Man and Cybernetics, SMC 2008, IEEE International Conference, Singapore, (2008) October 12-15.

[7] A. Barabasi and R. Albert, "Emergence of scaling in random networks", Science, vol. 286, no. 5439, (1999).

[8] Y. Hang, C. Guiran and W. Xingwei, "A cold-start recommendation algorithm based on new user's implicit information and multi-attribute rating matrix", Hybrid Intelligent Systems, HIS'09. Ninth International Conference, Shenyang, China, vol. 2, (2009) August 12-14.

[9] X. Lam, T. Vu, T. Le and A. Duong, "Addressing cold-start problem in recommendation systems", Proceedings of the 2nd international conference on Ubiquitous information management and communication, ACM, Suwon, Korea, (2008) January 31-February 01.

[10] D. Sun, Z. Luo, and F. Zhang, "A novel approach for collaborative filtering to alleviate the new item coldstart problem", Communications and Information Technologies (ISCIT), 2011 11th International Symposium, IEEE, Cordoba, Spain, (2011) November 22-24, pp. 402-406.

[11] P. Cremonesi and R. Turrin, "Analysis of cold-start recommendations in iptv systems", Proceedings of the third ACM conference on Recommender systems, ACM, New York, USA, (2009) October 22-25.

[12] V. Zanardi and L. Capra, "A scalable tag-based recommender system for new users of the social web, in Database and Expert Systems Applications", Edited Abdelkader Hameurlain, Stephen W. Liddle, KlausDieter Schewe and Xiaofang Zhou, Springer Berlin Heidelberg Publisher, (2011), pp. 542-557.

[13] M. Hameed, O. Al Jadaan and S. Ramchandram, "Information theoretic approach to cold start problem using genetic algorithms", Proceeding of the 2010 International Conference on Computational Intelligence and Communication Networks (CICN), Bhopal, India, (2010) November 26-28.

[14] J. He and W. Chu, "A social network-based recommender system (snrs)", Data Mining for Social Network Data, Edited Nasrullah Memon, Jennifer Jie Xu, David L.Hicks and Hsinchun Chen, Springer US Publishers, (2010), pp. 47-74.

[15] P. Victor, M. De Cock, C. Cornelis and A. Teredesai, "Getting cold start users connected in a recommender system's trust network", Computational Intelligence in Decision and Control, Madrid, Spain, vol. 1, (2008) September 21-24.

[16] L. Perez, B. Montes-Berges and M. del Rosario Castillo-Mayen, "Boosting social networks in social network-based recommender system", The 11th International Conference on Intelligent Systems Design and Applications (ISDA), IEEE, (2011).

[17] R. Hanneman and M. Riddle, Introduction to social network methods, (2005). 
[18] X. Li and L. Chen, "Recommendations based on network analysis", Advanced Computer Science and Information System (ICACSIS), 2011 International Conference on, IEEE, Jakarta, Indonesia, vol. 1, (2008) September 21-24.

[19] J. Herlocker, J. Konstan, A. Borchers and J. Riedl, "An algorithmic framework for performing collaborative filtering", Proceedings of the 22nd annual international ACM SIGIR conference on Research and development in information retrieval, ACM, California, USA, (1999) August 15-19.

[20] L. Admamic and E. Adar, "Friends and neighbors on the web", Social networks, vol. 3, no. 25, (2003).

[21] A. Anglade, M. Tiemann and F. Vignoli, "Complex-network theoretic clustering for identifying groups of similar listeners in p2p systems", Proceedings of the 2007 ACM conference on Recommender systems, Minneapolish, Minnesota, USA, (2007) October 19-20.

[22] F. Fouss, A. Pirotte, J. Renders and M. Saerens, "Random-walk computation of similarities between nodes of a graph with application to collaborative recommendation", IEEE Transactions on Knowledge and Data Engineering, NJ, USA, vol. 19, no. 3, (2007) March.

[23] Z. Huang, H. Chen and D. Zeng, "Applying associative retrieval techniques to alleviate the sparsity problem in collaborative filtering", ACM Transactions on Information Systems (TOIS), vol. 22, no. 1, (2004).

[24] Sanjiv Sharma and R. K. Gupta, "Improved BSP Clustering Algorithm for Social Network Analysis", IJGDC (2010) September, vol. 3, no. 3, pp. 67-76.

[25] L. Liu, K. Kim and W. Sun, "An Empirical Research of Factors Affecting Collaborative Decision Making System”, IJHIT, vol. 5, no. 4, (2012) October, pp. 1-10.

\section{Authors}

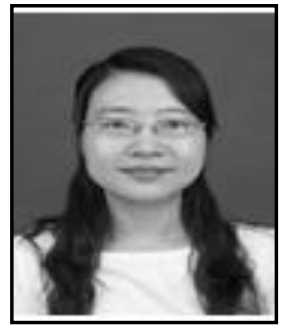

Yang Yujie received the bachelor's degree in Communication Engineering from The PLA Information Engineering University and the master's degree in Communication Engineering from Shanghai Normal University, China in 2002 and 2009 respectively. She is currently working in school of computer and information technology, Henan Normal University. She is currently researching on Wireless Communication, Distributed Network and Wireless Resource Management.

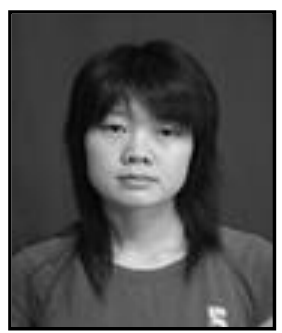

sZhang Huizhi received the bachelor's degree in Electronic Science and Technology from Zhengzhou University and the master's degree in photology from Anhui Optical Precision Machinery Research Institute, Chinese Academy of Sciences, China in 2003 and 2006 respectively. She is working in school of computer and information technology, Henan Normal University. She is currently researching on Smart Antenna and Adaptive Signal Processing.

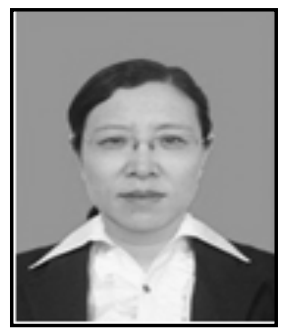

Wang Xianfang received the $\mathrm{Ph} . \mathrm{D}$. degree in Communication and Control Engineering from Jiangnan University, Wuxi, Jiangsu Province, China, in 2010. Currently, she is an Associate Professor of Computer Science and Communication Engineering in Department of Computer and Information Technology, Henan Normal University. Her research interests include machine learning, modeling and optimized controlling of biotechnical process. 
International Journal of $u$ - and e- Service, Science and Technology Vol.6, No.6 (2013) 